Solution by L. Carlitz.

$$
\begin{aligned}
& x^{13}+x+90 \equiv x^{13}+x-1 \\
& x^{13}+x-1 \equiv\left(x-\frac{1}{2}\right)\left\{\left(x-\frac{1}{2}\right)^{12}+1\right\}
\end{aligned}
$$

Since

$$
y^{12}+1 \equiv\left(y^{2}-2\right)\left(y^{2}-5\right)\left(y^{2}-6\right)\left(y^{2}-7\right)\left(y^{2}-8\right)\left(y^{2}-11\right)
$$

(the numbers $2,5,6,7,8,11$ are the quadratic non-residues $(\bmod 13))$ we get the quadratic factors

$$
x^{2}-x+c, c= \pm 5,4,3,2,-1
$$

Next, if $f(x)=x^{13}+x-1$, then

$$
f\left(\frac{1}{2}\right) \equiv f^{\prime}\left(\frac{1}{2}\right) \equiv 0
$$

so that $f(x)$ is divisible $(\bmod 7)$ by $\left(x-\frac{1}{2}\right)^{2}$, which is congruent to $x^{2}-x+2$. Since this polynomial occurs among the quadratics ( $\bmod 13)$ found above, it is a likely candidate. By division we find that

$$
\begin{aligned}
x^{13}+x+90 & =\left(x^{2}-x+2\right)\left(x^{11}+x^{10}-x^{9}-3 x^{8}-x^{7}+5 x^{6}\right. \\
& \left.+7 x^{5}-3 x^{4}-17 x^{3}-11 x^{2}+23 x+45\right)
\end{aligned}
$$

It would be interesting to know whether the second factor is irreducible. (Also solved by the proposer.)

\title{
SEQUENCE AND SERIES TRANSFORMATIONS
}

$$
\text { M. S. Macphail }
$$

The summability methods

$$
\text { A: } t_{n}=\sum_{k=0}^{\infty} a_{n k} s_{k}, \quad \text { B: } T_{n}=\sum_{k=0}^{\infty} b_{n k} u_{k} \text {, }
$$

where $b_{n k}=a_{n k}+a_{n, k+1}+\ldots$, are regarded as the sequence to-sequence and series-to-sequence forms of the same method, and if $s_{k}=u_{0}+\ldots+u_{k}$, we speak of the series $\sum u_{k}$ or the 
sequence $\left\{s_{k}\right\}$ indifferently, as summable $A$ or $B$. We have by partial summation

(1) $b_{n o} u_{0}+\ldots+b_{n k} u_{k}=a_{n o} s_{0}+\ldots+a_{n, k-1} s_{k-1}+b_{n k} s_{k}$;

so in order that $B \supset A$ (every $A$-summable sequence is $B$-summable to the same sum) it is necessary and sufficient that $\lim _{n} \lim _{k} b_{n k} s_{k}=0$ for every $A$-summable sequence $\left\{s_{k}\right\}$, and in order that $A \supset B$ it is necessary and sufficient that the same holds for every $B$-summable $\left\{s_{k}\right\}$.

The purpose of this note is to give simple sufficient conditions depending on the coefficients $b_{n k}$ alone.

THEOREM 1. In order that $B \supset A$, it is sufficient that for each $n=0,1, \ldots$ there is a positive constant $R_{n}$ such that $\left|1-b_{n, k+1} / b_{n k}\right|>R_{n}(k=0,1, \ldots)$.

Proof. For $B>A$, it is plainly sufficient that $T_{n}$ exists and equals $t_{n}$, for every $\left\{s_{k}\right\}$ such that $t_{n}$ exists; or, from (1), that $A_{n}^{*} \supset A_{n}$, where

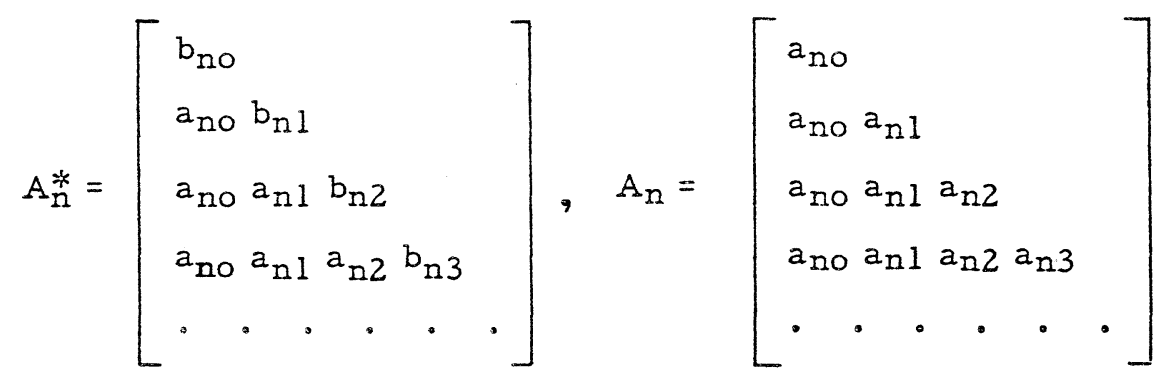

We easily find that $A_{n}^{*} A_{n}^{-1}$ has for its $k$-th row

$$
\left(0,0, \ldots, 0,1-b_{n k} / a_{n k}, b_{n k} / a_{n k}\right)
$$

Applying the Toeplitz conditions for regularity, we have at once that the column limits are zero and the row-sum limit is 1 . The row-norm condition reduces to $\left|b_{n k} / a_{n k}\right|<M_{n}$, which is equivalent to the condition stated in the theorem.

THEOREM 2. In order that $A \supset B$, it is sufficient that for each $n=0,1, \ldots$ there is a constant $M_{n}$ such that

(2) $\left|b_{n, k+1}\right| \sum_{r=0}^{k}\left|b_{n, r+1}^{-1}-b_{n r}^{-1}\right|<M_{n} \quad(k=0,1, \ldots)$, and $\lim _{\mathrm{k}} \mathrm{b}_{\mathrm{nk}}=0$. 
This may be proved by a similar method, after writing (1) in the modified form

$$
\begin{aligned}
& a_{n o} s_{0}+a_{n l} s_{1}+\ldots+a_{n k} s_{k} \\
= & b_{n o} u_{0}+b_{n l} u_{1}+\ldots+b_{n k} u_{k}-b_{n, k+1} s_{k} \\
= & \left(b_{n o}-b_{n, k+1}\right) u_{0}+\left(b_{n 1}-b_{n, k+1} u_{1}+\ldots+\left(b_{n k}-b_{n, k+1}\right) u_{k} .\right.
\end{aligned}
$$

Or we may use a theorem of Kronecker [1, p. 129-130], to show that $\lim _{\mathrm{k}} b_{\mathrm{nk}} \mathrm{s}_{\mathrm{k}}=0$.

THEOREM 3. In order that $A \supset B$, it is sufficient that for each $n=0,1, \ldots$ there is a constant $C_{n}\left(0<C_{n}<1\right)$, such that $\left|b_{n, k+1} / b_{n k}\right|<C_{n}(k=0,1, \ldots)$. For real $b_{n k}$ it is sufficient that for each $n, b_{n k} \rightarrow 0$ monotonically from a certain $k$ on.

Proof. The second condition is obviously sufficient for (2). For the first, we observe that

$$
\left|b_{n, k+1}\right| \sum_{r=0}^{k}\left|b_{n, r+1}^{-1}-b_{n}^{-1}\right|<2\left|b_{n, k+1}\right| \sum_{r=0}^{k+1}\left|b_{n r}^{-1}\right| .
$$

Denoting the right hand side by $2 B_{n k}$, we find

$$
B_{n, k+1}=\left|b_{n, k+2} / b_{n, k+1}\right| B_{n k}+1 \text {, }
$$

whence we see inductively that $B_{n k}$ is bounded, under our hypothesis.

We may illustrate with the well-knowr "circle method":

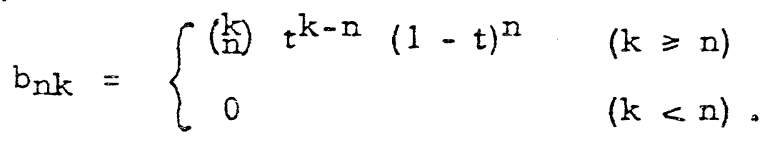

This is in the customary series-to-series form. We easily obtain from Theorems 1 and 3 the known results [2, p.549; 3, p. 141] that (with $a_{n k}=b_{n k}-b_{n, k+1}$ ) we have $B \supset A$ for all $t \neq 1$ and $A>B$ for $|t|<1$; here $A$ is a sequence-to-series method which is equivalent to the corresponding sequence-tosequence method. It is easily proved [2] that the condition $|t|<1$ is necessary for $A>B$. 


\section{REFERENCES}

1. K. Knopp, Theory and Application of Infinite Series (London, 1928).

2. P. Vermes, Series to series transformations and analytic continuation by matrix methods. Amer. J. Math. 71 (1949), $541-562$.

3. K. Zeller, Theorie der Limitierungsverfahren (Berlin, 1958).

Carleton University 